\title{
Evaluation of oxidative stability of mayonnaise containing poly $\varepsilon$-caprolactone nanoparticles loaded with thyme essential oil
}

\author{
Rhaysa Beatriz dos Passos', Giovana Carolina Bazzo ${ }^{1}$, Aline da Rosa Almeida ${ }^{1}$, \\ Carolina Montanheiro Noronha ${ }^{1}$, Pedro Luiz ManiqueBarreto*,1
}

\author{
${ }^{1}$ Department of Food Science and Technology, Federal University of Santa Catarina,Florianópolis, SC, Brazil
}

\begin{abstract}
Suspensions of poly $\varepsilon$-caprolactone (PCL) nanoparticles loaded with thyme essential oil were prepared as a natural antioxidant in mayonnaise. Mean particle size was $204.9 \pm 2.7$ and $240.0 \pm 5.5 \mathrm{~nm}$ respectively for nanoparticles prepared with PCL alone (NP-C) and for those loaded with thyme essential oil (NP$\mathrm{T})$. The polydispersity index indicated a homogeneous distribution of all particles, with no significant difference between NP-C and NP-T samples. The nanoparticles showed a large negative charge evidenced by zeta potential rates, indicating high physical stability. The use of PCL as a polymer provided high encapsulation efficiency for thyme essential oil (91.15 $\pm 2.12 \%)$. DPPH (2,2-diphenyl-1-picrylhydrazyl) method determined $\mathrm{IC}_{50}$ rates were $476.4 \pm 33.6$ and $483.5 \pm 20.4 \mu \mathrm{g} \mathrm{mL}{ }^{-1}$ respectively for unencapsulated oil and for NP-T, evidencing pronounced antioxidant activity. NP-C, NP-T and synthetic antioxidant butylhydroxytoluene (BHT) were applied to samples of mayonnaise and their oxidative stability evaluated for eight days in an oven at $63 \pm 3^{\circ} \mathrm{C}$. Results of hydroperoxide value (HP) and thiobarbituric acid reactive substances (TBARS) showed that NP-T had a similar performance as synthetic antioxidant BHT in the prevention of mayonnaise lipid oxidation.
\end{abstract}

Keywords: Nanoparticles.Poly $\varepsilon$-caprolactone. Thyme Essential Oil. Mayonnaise. Oxidative stability. Antioxidants/adverse effects. Thymus Plant. Aromatherapy/utilization. Butylated Hydroxytoluene/ analysis. Oxidation/prevention \& control.

\section{INTRODUCTION}

There is currently a world wide trend to substitute synthetic compounds for those of natural origin, in nutrition and in other areas. Essential oils extracted from different plants may be an alternative for use in the nutrition area since, depending on the composition of the essential oil, they have antimicrobial, antifungal, antioxidant and other properties (Yuan, Chen, Li, 2016; Tao et al., 2014; Chatterjee, Bhattacharjee, 2013). Due to this trend, the use of synthetic antioxidants, such as butylhydroxytoluene (BHT) and butylhydroxyanisole (BHA) have been avoided and replaced by naturally occurring antioxidants(Dogu-Baykut, Gunes, Decker, 2014).

Thyme essential oil features well-known antioxidant activities and may be used as a natural antioxidant, besides

\footnotetext{
*Correspondence: P. L. M. Barreto. Department of Food Science and Technology, Federal University of Santa Catarina. Rodovia Admar Gonzaga, 1346, Itacorubi, 88034-001, Florianópolis, SC, Brazil. Phone: + 55 (48) 37215368. E-mail: pedro.barreto@ufsc.br
}

providing food with the oil's typical special flavor. Its antioxidant effect is due to great amounts of phenolic compounds, especially thymol and carvacrol, in its composition (Walker et al., 2017). However, the use of essential oils in food may present several problems, such as degradation by exposure to light, heat or oxygen; difficulty in application (due to its limited aqueous solubility);rapid loss by volatilization; and possible changes in the product's organoleptic characteristics. In fact, that is why the nano/microencapsulation of essential oils is suggested as an alternative to their direct application on foods (Gonçalves et al., 2017; Chatterjee, Bhattacharjee, 2013). Research works described in the literature revealed the encapsulation of thyme essential oil in microspheres, employing different wall materials: $\beta$-cyclodextrin (Chatterjee, Bhattacharjee, 2013), alginate (Benavides et al., 2016) and gelatin/arabic gum (Gonçalves et al., 2017). In addition, other assays describe its incorporation into nanoparticles of high methoxyl pectin (Guerra-Rosas et al., 2016), sodium alginate (Salvia-Trujillo et al., 2015) and chitosan (Sotelo-Boyás et al., 2017). 
Nanoemulsions, which consist of small droplets $(<200 \mathrm{~nm})$ dispersed in water, are particularly suitable for encapsulating hydrophobic bioactive agents, such as essential oils. They have gained popularity because of their ease in preparation and handling and their high physical stability. Food, food supplements and pharmaceutical industries are developing nanoemulsion-based delivery systems to encapsulate, protect and control the release of various hydrophobic bioactive agents (Walker et al., 2017).

Current study aimed at nano-encapsulating thyme oil, with known antioxidant action, using PCL as a wall material, a hydrophobic, non-toxic, biodegradable and biocompatible aliphatic polyester, widely used in the pharmaceutical and food area (Peña et al., 2016). As mentioned above, nano-encapsulation of essential oils has several advantages over the use of unencapsulated oils. Further, the thyme oil-loaded PCL nanoparticles were applied to a food product (mayonnaise) to evaluate their activity as a natural antioxidant and to compare with the synthetic antioxidant BHT.

\section{MATERIALS AND METHODS}

\section{Materials}

PCL(Mw 70.000-90.000) and DPPH reagent were purchased from Sigma Aldrich (St Louis, USA) and soy lecithin (LIPOID S 75) was obtained from Lipoid (Ludwigshafen, Germany). Thyme essential oil and raw materials for the preparation of mayonnaise were kindly donated by Duas Rodas Industrial (Jaraguá do Sul, Brazil). The poloxamer surfactant (Pluronic ${ }^{\circledR}$ F68) was donated by BASF Chemicals Company (USA). All other reagents used were of analytical grade, with the exception of methanol for chromatographic analysis (Sigma Aldrich, St Louis, USA).

\section{Preparation of PCL nanoparticles containing thyme essential oil}

PCL nanoparticles containing thyme essential oil(NP-T) were prepared by nanoprecipitation, following methodology by Noronha et al. (2013), with some adaptations. PCL was first solubilized in $2.0 \mathrm{~mL}$ acetone and added to $10 \mathrm{~mL}$ of a lecithin solution in acetone/ ethanol $(60: 40, \mathrm{v} / \mathrm{v})$, followed by the addition of thyme oil (300 mg).The solution was then dripped into $25 \mathrm{~mL}$ of an aqueous phase containing $1 \%(\mathrm{w} / \mathrm{v})$ hydrophilic surfactant (Pluronic ${ }^{\circledR} \mathrm{F} 68$ ), under magnetic stirring at $25{ }^{\circ} \mathrm{C}$. The aqueous phase became immediately milky due to the formation of the nanoparticle suspension. The organic solvents were then eliminated by evaporation and the final volume adjusted to $25 \mathrm{~mL}$. The resulting suspension was filtered through an $8 \mu \mathrm{m}$ pore-size filter paper (Millipore, USA) and stored under refrigeration, protected from light. Nanoparticles were also prepared without the addition of the essential oil, called control nanoparticles (NP-C).

\section{Characterization of PCL nanoparticles containing thyme essential oil}

Mean particle size (PS), polydispersity index (PDI) and zeta potential $(\zeta)$ of the thyme oil-loaded PCL nano capsules were determined by dynamic light scattering using a ZetasizerNanoSeries (Malvern Instruments, Worcestershire, UK). Measurements were performed in triplicate at $25^{\circ} \mathrm{C}$ after appropriate dilution $(1: 10, \mathrm{v} / \mathrm{v})$ of the nanocapsule suspensions in Milli- $\mathrm{Q}^{\circledR}$ water.

The encapsulation efficiency (EE\%) has been defined as the difference between total thyme oil content in nanoparticle suspension and the free thyme oil content obtained after the separation of the loaded nanoparticles from the aqueous medium by ultrafiltration/centrifugation procedure using Amicon Ultra-Centrifugal Filters with Ultracel 30k membrane (Millipore, Ireland). Samples were centrifuged at $5600 \mathrm{xg}$ for $30 \mathrm{~min}$ at $20^{\circ} \mathrm{C}$. The free thyme oil concentration in the supernatant was then determined by high performance liquid chromatography with Shimadzu chromatograph (LC-20AT, Shimadzu, Kyoto, Japan), according to methodology described by Rosa et al. (2015). EE\% was then calculated by Equation 1, considering the fraction of free (unencapsulated) oil and the fraction initially present in the nanoparticles.

$$
E E \%=100-\left(\frac{\text { free oil concentration }}{\text { total oil concentration }}\right) \quad \text { Equation } 1
$$

The morphology of thyme oil-loaded PCL nanoparticles was examined with a transmission electron microscope (TEM). TEM micrographs were recorded with JEOL JEM-1011microscope (Tokyo, Japan). The concentrated nanoparticles suspension was first diluted in water at $1 / 3$ before directly deposited on carbon-coated 200 mesh copper grids. Samples were observed after drying.

\section{Determination of antioxidant activity (AA)}

Antioxidant activity was determined by the capacity of samples to inhibit the radical DPPH, according to method by Afoulous et al. (2013), with modifications. 
Samples (free thyme oil and NP-T) were diluted at concentrations ranging between 90 and $470 \mu \mathrm{g} \mathrm{mL}^{-1}$ using methanol as solvent. Further, $1.5 \mathrm{~mL}$-aliquots from each dilution were transferred into test tubes, followed by the addition of $1.5 \mathrm{~mL}$ of the reagent (DPPH $0.2 \mathrm{mM}$ ).A blank was also prepared by replacing the dilution with the same volume of methanol. After $30 \mathrm{~min}$, absorbance (Abs) at $520 \mathrm{~nm}$ was determined on a BEL-Photonics spectrophotometer SP 2000 (Piracicaba, SP,Brazil). Assay was performed in triplicate. The percentage of AA was calculated by Equation 2 and results were expressed as $\mathrm{IC}_{50}$ rates, calculated by linear regression, where $\boldsymbol{x}$ axis represented the tested oil concentrations and $y$ axis the percentage of antioxidant activity.

$$
A A=\left[\frac{(\text { Abs blank }- \text { Abs sample })}{\text { Abs blank }}\right] \times 100
$$

Equation 2

\section{Preparation and evaluation of mayonnaise oxidation}

The mayonnaise, produced in a blender Philips Walita (São Paulo, Brazil), contained the following ingredients: soybean oil without antioxidant (65.0\%), filtered water (20.95\%), alcohol vinegar (7.4\%), dehydrated egg (2.3\%), sodium chloride $(2.0 \%)$, sugar $(1.5 \%)$, modified starch $(0.5 \%)$, xanthan gum $(0.1 \%)$, potassium sorbate $(0.1 \%)$, lactic acid $(0.1 \%)$ and concentrated lemon juice $(0.05 \%)$.

Three batches were produced: one batch contained NP-T $(0.01 \% \mathrm{w} / \mathrm{w})$; another batch contained the synthetic antioxidant BHT $(0.01 \% \mathrm{w} / \mathrm{w})$ (positive control); the third batch contained NP-C $(0.01 \% \mathrm{w} / \mathrm{w})$ (negative control). After preparation, each batch was packed in sealed glass vials, pasteurized and stored in an oven at $63 \pm 3^{\circ} \mathrm{C}$ (Schaal Oven Test). During an eight-day period, the samples were periodically analyzed for hydroperoxide value and TBARS.

HP was determined by titration, following the official methodology of the American Oil Chemists' Society (Cd 8b-90, AOCS, 2017). Prior to analysis, the mayonnaise samples were transferred to Falcon tubes and frozen in a freezer for 24 hours. After thawing, the oil phase was separated by centrifugation at $6000 \mathrm{rpm}$ for 5 min and then analyzed.

TBARS assay was performed following Altunkaya et al. (2013), with modifications. Mayonnaise samples were frozen/thawed, as previously described, to separate the oil phase. Further, approximately $50 \mathrm{mg}$ of oil were transferred to a test tube; $10 \mathrm{mLn}$-butanol were added and homogenized. A $2 \mathrm{~mL}$ aliquot was transferred to another test tube, followed by the addition of $5 \mathrm{~mL}$ thiobarbituric acid solution $(0.2 \% \mathrm{w} / \mathrm{v}$, in n-butanol). Samples were incubated at $95^{\circ} \mathrm{C}$ for 1 hour and absorbance determined at $532 \mathrm{~nm}$ with spectrophotometer BEL-Photonics SP 2000 (Piracicaba, SP,Brazil). TBA value $(1 / \mathrm{mg})$ was then calculated (Equation 3), where $\boldsymbol{A s}$ is the sample absorbance; $\boldsymbol{A} \boldsymbol{b}$ is the blank absorbance; $\boldsymbol{m}$ is the mass of oil sample (mg).

$T B A R S=\frac{[50 \times(A s-A b)]}{m}$

Equation 3

ANOVA test evaluated significant difference between samples, coupled to Tukey's test at $95 \%$ significance.

\section{RESULTS AND DISCUSSION}

\section{Preparation and characterization of $\mathrm{PCL}$ nanoparticles containing thyme essential oil}

Mean particle size (Table I) was204.9 \pm 2.7 and $240.0 \pm 5.5 \mathrm{~nm}$ respectively for nanoparticles prepared without and with thyme essential oil (NP-C and NP-T). No significant difference was observed between the mean diameter of NP-C nanoparticles and those containing thyme oil (NP-T) ( $>>0.05)$. Noronha et al. (2013) prepared nanoparticles of PCL containing $\alpha$-tocopherol by the nanoprecipitation method and obtained particles averaging in size between 184 and $219 \mathrm{~nm}$, corroborating results in current study. In the case of the nanoprecipitation method, factors such as the nature and concentration of the polymer in the organic phase, solvent polarities, the nature and ratio of the internal/external phases and the nature and concentration of the surfactant were essential to determine the size of the nanoparticles.

PDI is a parameter used to verify homogeneity in particle size distribution. PDI values in Table I indicate a homogeneous distribution of all particles, with no significant difference between NP-C and NP-T samples. PDI values below 0.2 indicate uniformity among oil droplet sizes or monomodal distributions and therefore better stability, whereas rates close to 1 indicate heterogeneous or multimodal distribution (Guerra-Rosas et al., 2016).

Nanoparticles showed a large negative charge evidenced by zeta potential values (Table I). Since the stabilizer used (Pluronic ${ }^{\circledR}$ F68) is a non-ionic surfactant, the negative zeta potential values are caused by the lecithin, with negatively charged carboxyl groups in free fatty acids (Hatanaka et al., 2010). In theory, if the absolute value of zeta potential is below $30 \mathrm{mV}$, the 
TABLE I - Results for mean particle size (Z-ave), polydispersity index (PDI), zeta potential ( $\zeta$ ) and encapsulation efficiency (EE\%) for PCL nanoparticles

\begin{tabular}{lcccc}
\hline & Z-ave (nm) & PDI & $\zeta(\mathbf{m V})$ & EE\% \\
\hline NP-C & $204.9 \pm 2.7$ & $0.21 \pm 0.01$ & $-50.95 \pm 1.62$ & - \\
NP-T & $240.0 \pm 5.5$ & $0.17 \pm 0.02$ & $-51.00 \pm 0.84$ & $91.15 \pm 2.12$ \\
\hline
\end{tabular}

nanoemulsion exhibits a weak stability (agglomerationflocculation), whereas for absolute values higher than 30 $\mathrm{mV}$, the nanoemulsions are presumed to be stable due to electrostatic repulsion(Guerra-Rosas et al., 2016). The zeta potential values obtained for NP-C and NP-T indicate high physical stability.

With respect to EE\% result, it may be verified that most of the oil $(91.15 \pm 2.12 \%)$ remained inside the particles and showed that the nanoprecipitation method and the use of PCL as a polymer provided high encapsulation efficiency for the thyme essential oil. Sotelo-Boyás et al. (2017) obtained EE\% rates between 68 and $72 \%$ when they encapsulated thyme oil in chitosan nanoparticles.

The occurrence of spherical and isolated particles without agglomeration may be observed in the micrographof NP-T particles obtained by TEM (Figure 1).

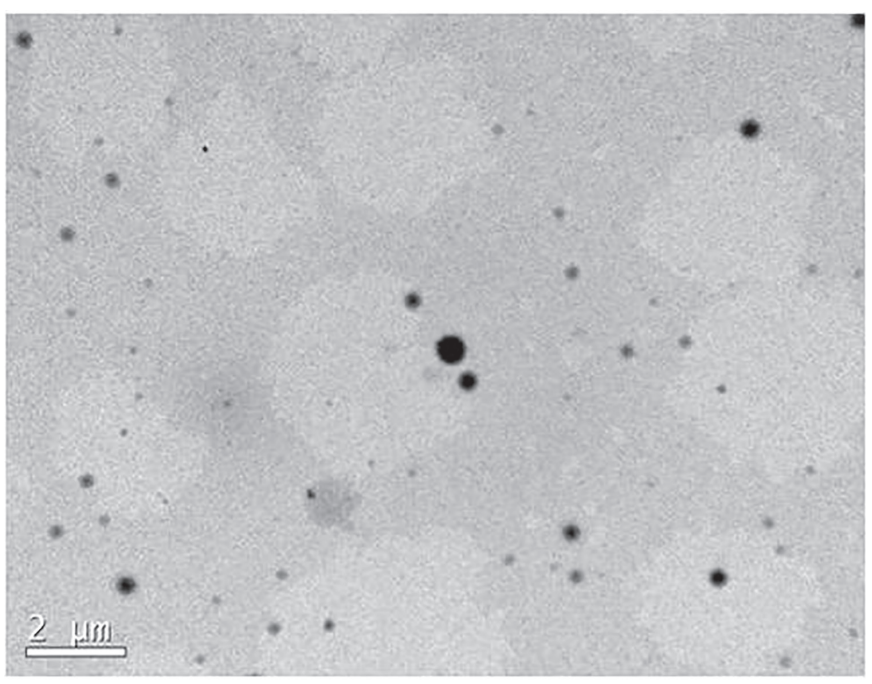

FIGURE 1 - TEM micrograph of PCL nanoparticles containing thyme essential oil.

\section{Determination of antioxidant activity}

The AA of unencapsulated thyme essential oil and NP-T were evaluated by DPPH scavenging activity test and results were expressed as $\mathrm{IC}_{50}$, or rather, the sample concentration required to cause $50 \%$ inhibition of $\mathrm{DPPH}$ reagent. Consequently, the higher the $\mathrm{IC}_{50}$ value, the lower the antioxidant activity of the sample tested.
Rates were $476.4 \pm 33.6$ and $483.5 \pm 20.4 \mu \mathrm{g} \mathrm{mL}^{-1}$ for the unencapsulated oil and for NP-T, respectively, and indicated that the AA of the thyme oil was not affected by the nanoencapsulation process.

Tohidi, Rahimmalek, Arzani (2017) evaluated the AA of essential thyme oils obtained from different species of Thymus and found $\mathrm{IC}_{50}$ rates within the range of 273 to $693 \mu \mathrm{g} \mathrm{mL}^{-1}$,corroborating those obtained in current study. According to the literature, differences in the composition of essential oil may exist due to plant culture (soil, climate), season and extraction method. Further, the amount of thymol present in thyme oil varied between approximately $6.8 \%$ and $64.5 \%$ (Lemos et al., 2017). Thyme essential oil in current assay presented the following major compounds in its composition: p-cymene $(26.38 \%)$, thymol (47.36 \%) and carvacrol (3.24\%) (results obtained by gas chromatography, data not shown), the latter two being the main responsible for its antioxidant activity (Walker et al., 2017).

\section{Study on the stability oxidation of mayonnaise enriched with nanoparticles}

Many lipid-containing food products, such as mayonnaise, are very susceptible to oxidative deterioration especially if they contain high amounts of polyunsaturated fatty acids. The oxidation of lipids is a detrimental process that causes nutritional losses and development of undesirable flavor and color. As a consequence, food manufacturers are constantly searching for methods to prevent or, at least, to retard lipid oxidation in foods. Natural and synthetic antioxidants may decrease the rate of lipid oxidation by scavenging and reducing the activity of free radicals, thus inhibiting initiation and propagation reactions (Dogu-Baykut, Gunes, Decker, 2014).

In current study, PCL nanoparticles loaded with thyme essential oil were applied to a mayonnaise sample and its antioxidant action was compared to the synthetic antioxidant BHT. Samples were maintained at a temperature of $63 \pm 3^{\circ} \mathrm{C}$ for 8 days (Schaal Oven Test). The Schaal Oven Test, involving heating the sample at $60-70^{\circ} \mathrm{C}$ until the appearance of the first signs of oxidation, provides a good correlation with assessment under normal storage 
Evaluation of oxidative stability of mayonnaise containing poly $\varepsilon$-caprolactone nanoparticles loaded with thyme essential oil

TABLE II - HP (meq hydroperoxides per $\mathrm{kg}$ oil) and TBARS $(1 / \mathrm{mg})$ values of mayonnaise samples stored at $63^{\circ} \mathrm{C}$ for 8 days

\begin{tabular}{cccc}
\hline & NP-C & NP-T & BHT \\
\hline HP (day 0) & $6.8 \pm 0.4$ & $4.9 \pm 0.5$ & $5.9 \pm 0.3$ \\
(day 1) & $7.2 \pm 0.6$ & $6.9 \pm 0.2$ & $7.6 \pm 0.0$ \\
(day 3) & $12.4 \pm 0.8$ & $12.7 \pm 0.5$ & $13.3 \pm 0.3$ \\
(day 6) & $16.9 \pm 0.7$ & $7.2 \pm 0.5$ & $9.6 \pm 0.8$ \\
(day 8) & $11.9 \pm 0.8$ & $3.1 \pm 0.2$ & $7.5 \pm 0.6$ \\
\hline TBARS (day 0) & $0.088 \pm 0.010$ & $0.087 \pm 0.011$ & $0.079 \pm 0.021$ \\
(day 1) & $0.124 \pm 0.003$ & $0.106 \pm 0.024$ & $0.122 \pm 0.020$ \\
(day 3) & $0.142 \pm 0.021$ & $0.108 \pm 0.022$ & $0.116 \pm 0.013$ \\
(day 6) & $0.178 \pm 0.006$ & $0.166 \pm 0.020$ & $0.173 \pm 0.011$ \\
(day 8) & $0.080 \pm 0.010$ & $0.075 \pm 0.007$ & $0.079 \pm 0.008$ \\
\hline
\end{tabular}

conditions (Silva, Borges, Ferreira, 1999). The oxidation of mayonnaise was monitored by HP and TBARS assays.

HP results (Table II) showed that there was a significant increase in hydroperoxides present in all analyzed samples (NP-C, BHT and NP-T) on the third day of storage. Results also indicated that mayonnaise samples already showed signs of oxidation after three days under oven storage. Hydroperoxides, primary products of lipid oxidation, are the first compounds to be formed as soon as the oxidation process begins (Silva, Borges, Ferreira, 1999). However, up to the third day of storage, no statistically significant differences $(p>0.05)$ were observed between the HP values of the three samples. After six days of storage, the sample without any addition of antioxidant (NP-C) continued to show increased HP values, indicating that, in this sample, a higher degree of lipid oxidation occurred when compared to the others. During the same period (six days), HP values for BHT and NP-T decreased, which was already expected. Hydroperoxides are unstable compounds that produce a number of secondary products such as alkanes, alcohols, aldehydes and acids (Li et al., 2014).

Malonaldehyde, unlike hydroperoxides, is one of the secondary products formed during lipid oxidation, quantified by TBARS assay, the results of which are shown in Table II. Samples with no antioxidant (NP-C) showed the highest TBARS rates, especially between the third and sixth day, and indicated the occurrence of higher amounts of malonaldehyde in the sample and, therefore, a higher degree of secondary oxidation.

\section{CONCLUSION}

The nanoprecipitation method and the use of PCL as a wall material provided nanoparticles containing high values of encapsulated thyme essential oil with good physical stability. In addition, the antioxidant activity of thyme oil was maintained after nanoencapsulation process. The application to mayonnaise samples, submitted to oven storage for eight days, showed that nanoparticles containing thyme essential oil presented similar performance to synthetic antioxidant BHT in the prevention of lipid oxidation.

\section{ACKNOWLEDGMENTS}

The authors would like to thank CNPq for its financial support and funding. Thanks are also due to DuasRodas Industrial for the donation of raw materials and essential oil.

\section{REFERENCES}

Afoulous S, Ferhout H, Raoelison EG, Valentin A, Moukarzel B,Couderc F, et al. Chemical composition and anticancer, antiinflammatory, antioxidant and antimalarial activities of leaves essential oil of Cedrelopsis grevei. Food Chem Toxicol. 2013;56: 352-62.

Altunkaya A, Hedegaard RV, Harholt J, Brimer L, Gökmen V,Skibsted LH. Oxidative stability and chemical safety of mayonnaise enriched with grape seed extract. Food Funct. 2013;4(11):1647-53.

AOCS. Official methods and recommended practices of the American Oil Chemists'Society. 7.ed. Champaign, Illinois: AOCS Press, 2017.

Benavides S, Cortés P, Parada J, Franco W. Development of alginate microspheres containing thyme essential oil using ionic gelation. Food Chem. 2016; 204:77-83. 
Chatterjee D,Bhattacharjee P. Comparative evaluation of the antioxidant efficacy of encapsulated and un-encapsulated eugenol-rich clove extracts in soybean oil: Shelf-life and frying stability of soybean oil. J Food Eng.2013;117(4):545-50.

Dogu-Baykut E, Gunes, G,Decker EA. Impact of shortwave ultraviolet (UV-C) radiation on the antioxidant activity of thyme (Thymus vulgaris L.). Food Chem. 2014;157:167-73.

Gonçalves ND, PenaFL, Sartoratto A, Derlamelina C, Duarte MCT, Antunes AEC, et al. Encapsulated thyme (Thymus vulgaris) essential oil used as a natural preservative in bakery product. Food Res Int. 2017;96:154-60.

Guerra-Rosas MI, Morales-Castro J, Ochoa-Martínez LA, Salvia-Trujillo L, Martín-Belloso O. Long-term stability of food-grade nanoemulsions from high methoxyl pectin containing essential oils. Food Hydrocolloids. 2016; 52:438-46.

Hatanaka J, Chikamori H, Sato H, Uchida S, Debari K,Onoue $\mathrm{S}$, et al. Physicochemical and pharmacological characterization of alpha-tocopherol-loaded nano-emulsion system. Int J Pharm. 2010; 396(1/2):188-93.

Lemos MF, Lemos MF, Pacheco HP, Guimarães AC, Fronza $\mathrm{M}$,Endringer DC, et al. Seasonal variation affects the composition and antibacterial and antioxidant activities of Thymus vulgaris. Ind Crops Prod. 2017;95:543-48.

Li CH, Kim HW, Li H, Lee DC, Rhee HI. Antioxidative effect of purple corn extracts during storage of mayonnaise. Food Chem. 2014;152:592-6.

Noronha CM, Granada AF, Carvalho SM, Lino RC, Maciel MVOB, Barreto PLM.Optimization of alpha-tocopherol loaded nanocapsules by the nanoprecipitation method. Ind Crops Prod. 2013; 50:896-903.
Peña JA, Gutiérrez SJ, Villamil JC, Agudelo NA, Pérez LD.Policaprolactone/polyvinylpyrrolidone/siloxane hybrid materials: synthesis and in vitro delivery of diclofenac and biocompatibility with periodontal ligament fibroblasts. Mater Sci Eng C Mater Biol Appl. 2016;58:60-9.

Rosa CG, Maciel MVOB, Carvalho SM, Melo APZ, Jummes B, Silva T, et al. Characterization and evaluation of physicochemical and antimicrobial properties of zein nanoparticles loaded with phenolics monoterpenes. Colloids Surfaces A: Physicochem EngAspects. 2015;481:337-44.

Silva FAM, Borges MFM,Ferreira MA. Métodos para avaliação do grau de oxidação lipídica e da capacidade antioxidante (Methods for the evaluation of the degree of lipid oxidation and the antioxidant activity. Quim Nova. 1999;22(1):94-103.

Sotelo-Boyás M, Correa-Pacheco Z, Bautista-Baños S,Gómez Y Gómez Y. Release study and inhibitory activity of thyme essential oil-loaded chitosan nanoparticles and nanocapsules against foodborne bacteria. Int J Biol Macromol. 2017; 103:40914.

Tohidi B, Rahimmalek M,Arzani A. Essential oil composition, total phenolic, flavonoid contents, and antioxidant activity of Thymus species collected from different regions of Iran. Food Chem. 2017; 220:153-61.

Yuan G, Chen X, Li D. Chitosan films and coatings containing essential oils: the antioxidant and antimicrobial activity, and application in food systems. Food Res Int. 2016; 89(pt 1):117-28.

Walker RM, Gumus CE, Decker EA,McClements, DJ. Improvements in the formation and stability of fish oil-in-water nanoemulsions using carrier oils: MCT, thyme oil, \& lemon oil. J Food Eng.2017;21:60-8.

Received for publication on $09^{\text {th }}$ March 2018 Accepted for publication on $16^{\text {th }}$ May 2018 